\title{
Matlab-Based Studying of 4G Digital Modulation Techniques over Various Channels
}

\author{
Yihuai Yang \\ School of Automatic Control and Mechanical Engineering, Kunming University, 650214, China \\ email: yyh_td@126.com
}

Keywords: 4G; QPSK; DQPSK; QAM; BER; SNR; SIMULINK.

Abstract: In this paper, a comparison studying of different 4G digital modulation techniques over AWGN channel, as well as Rayleigh and Rician fading channels were performed. Firstly, we investigated QPSK, DQPSK, 16-QAM and 64-QAM modulated signal constellations, and then we generated BER and SNR data for analysing different communication systems. At last, we used MATLAB SIMULINK to perform Monte Carlo simulate for 16-QAM system.

Original article, Published date: 2018-05-23

DOI: 10.23977/jeis.2018.31012

ISSN 2371-9532

https://www.clausiuspress.com/journal/JEIS.html

\section{Introduction}

There has been a dramatic move towards LTE 4G wireless communication systems and modulation is an important step of design and realize a good performance, reliable and robust 4G system.

Modulation is a process of mixing a signal with a sinusoid to produce a new signal[1]. The modulated signal has a great deal of benefits over an un-modulated one, due to the fact that the transition of analogue to digital modulation helping becoming wireless, improving data security, reducing noises, enhancing the quality of communication, adding information-carrying capacity, providing compatibility with digital data services as well as RF spectrum sharing to accommodate added services.

Extensive work about 4G modulation techniques have been carried out for many years[3-6]. However, most of them are focus on AWGN channel, and our knowledge of the radio channels 
encountered in mobile-to-mobile and relay-based systems are still inadequate. In this paper, we did comparative study of QPSK, DQPSK, QAM over different channels.

\section{Rayleigh and Rice Channels}

Digital scheme has become increasingly important to satisfy the operator requirement for network planning, optimization, interference and traffic calculation, as well as frequency planning and neighbors analyses. It convert the analogue signal to a digital signal and has tremendous benefits such as greater capacity to transmit large quantity of data with high noise immunity, and it can detect distinct transmission state at the receiver in a noisy environments[5]. So, the choice of digital modulation plays a crucial role in the development of Fourth Generation (4G) wireless communication systems. In LTE 4G systems, in order to get high bandwidth, depending on the speed needs, the modulation on each subcarriers can be quadrature phase-shift keying (QPSK), 16phase quadrature amplitude modulation (16QAM), or 64-state quadrature amplitude modulation (64QAM).

The AWGN channel is very simple and usually has been considered as a ideal condition to develop the basic system for performance evaluation. However, this channel model does not include any fading, which is dominant in the $4 \mathrm{G}$ channel. So, different from other papers, normally focus on AWGN channel, in this paper, we pay our attention on simulation studying different modulation based systems under Rice and Rayleigh fading channel.

\subsection{Rayleigh channels}

In multipath environments, when a narrowband signal transmitted over a randomly nonselective propagation path, it can be modeled as a zero-mean complex-valued Gaussian random process [8]:

$$
\mu(t)=\mu_{1}(t)+j \mu_{2}(t)
$$

where $\mu 1 \mu 2$ are real-valued Gaussian random processes with statistically uncorrelated. Then, the Rayleigh process [8] can be described by [1]:

$$
\zeta(t)=|\mu(t)|=\left|\mu_{1}(t)+j \mu_{2}(t)\right|
$$

A Rayleigh distribution is often observed when the overall multipath of the signals are LOS components.

\subsection{Rice channels}

The received signal of LOS component also can be denoted by a complex sinusoid form [1] :

$$
m(t)=m_{1}(t)+j m_{2}(t)=\rho e^{j\left(2 \pi f_{\rho} t+\theta_{\rho}\right)}
$$

where $\rho, f \rho, \theta \rho$ are the amplitude, the Doppler frequency and the phase, respectively.

Normally, the received multipath signals are the components of both LOS and NLOS signals, which makes it possible to introduce the complex Gaussian random process [1]:

$$
\mu_{\rho}(t)=\mu_{\rho 1}(t)+j \mu_{\rho 2}(t)=\mu(t)+m(t)
$$

with time-variant mean value $\mathrm{m}(\mathrm{t})=\mathrm{E}\{\mu \rho(\mathrm{t})\}$ to describe the signals at the receiver antenna. As a result, the Rice processes can be denoted by [1]:

$$
\xi(t)=\left|\mu_{\rho}(t)\right|=|\mu(t)+m(t)|
$$

\section{Modulation techniques}

The amplitude an phase information of a set of received signal points is commonly represented 
graphically on a constellation diagram.

\subsection{QPSK Modulation Schemes}

QPSK is a Double Side Band Suppressed Carrier (DSBSC) modulation scheme, which is a higher order of PSK. It is an extension of PSK. Instead of one, it sends two bits of digital information at a time, which represents $00,01,10$, or 11 . In this way, it could carry double information using the same bandwidth, so more bandwidth efficient [10].

The QPSK signal is expressed mathematical as[11]:

$$
\begin{aligned}
& s_{q p s k}(t)=\sqrt{\frac{2 E_{s}}{T_{s}}} \cos \left(2 \pi f_{c} t+(2 n-1) \frac{\pi}{4}\right), n=1,2,3,4 \\
& \phi_{1}(t)=\sqrt{\frac{2}{T_{s}}} \cos \left(2 \pi f_{c} t\right) \quad \phi_{2}(t)=\sqrt{\frac{2}{T_{s}}} \sin \left(2 \pi f_{c} t\right)
\end{aligned}
$$

where $T_{s}$ is the symbol period, $E_{s}$ is the energy per symbol.

The probability of bit error of QPSK in Additive White Noise Gaussian Noise (AWGN) is [5]:

$$
P_{e, \text { QPSK }}=Q\left[\sqrt{\frac{2 E_{b}}{N_{o}}}\right]
$$

where $E_{b}$ is energy per bit and $N_{0}$ is the noise power spectral density. $Q$ is the $Q$ function denoted by $Q(x)$.

$$
Q(x)=\frac{1}{\sqrt{2 \pi}} \int_{x}^{\infty} e^{-\left(\frac{t^{2}}{2}\right)} d t, \quad \int a^{x} d x=\frac{a^{x}}{\ln a}+C
$$

\subsection{QAM Modulation Schemes}

Quadrature conveys two analog message signals, or two digital bit streams, by modulating the amplitudes of two carrier waves, using the amplitude-shift keying (ASK) digital modulation scheme or amplitude modulation (AM) analog modulation scheme[11].

The QAM signal is expressed mathematical as[11]:

$$
s_{Q A M}(t)=\operatorname{Re}\left\{[I(t)+i Q(t)] e^{i 2 \pi f_{0} t}\right\}
$$

where $i^{2}=-1, I(t)$ and $Q(t)$ are the modulation signals, $f_{0}$ is the carrier frequency and $\operatorname{Re}\{\}$ is the real part. The average probability of error in an AWGN for M-ary QAM can be represented as [5]:

$$
P_{e} \cong 4\left(1-\frac{1}{\sqrt{M}}\right) Q\left(\sqrt{\frac{2 E_{\min }}{N_{o}}}\right)
$$

where $E_{\min }$ is the energy of the signal with the lowest amplitude.

\section{Simulation Results and Discussion}

Constellation Diagram is a graphical representation of the complex envelope of each possible signal. The distance between signals on a constellation diagram relates to how different the modulation waveforms are and how well a receiver can differentiate between them when random noise is present. In this section, we simulation studied different modulation techniques which have 
been used in LTE 4G systems, in Rayleigh fading channel.

Figure. 1. shows the QPSK constellation diagram under Rayleigh fading channel. In the diagram we can see that two bits per symbol with a minimum phase separation of $\pi / 2$. And we also simulated the DQPSK modulation situation, see Figure 2. In Figure 3, we give the Matlab simulation setup for 16-QAM modulation under multipath Rayleigh and Rician fading channels, separately. Other modulation techniques simulation setup are similar, just need to change the modulation block. The 16-QAM and 64-QAM constellation diagrams are show in Figure 4 and Figure 5, separately.

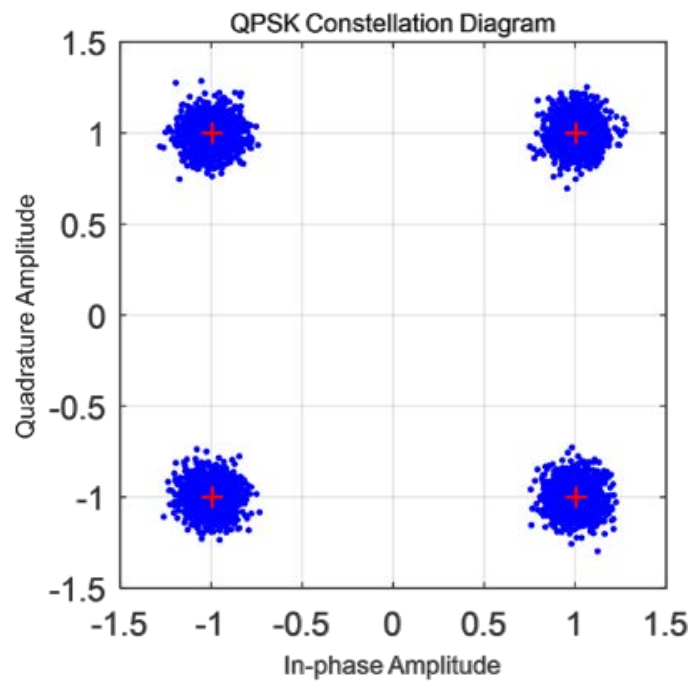

Figure 1. QPSK Constellation Diagram

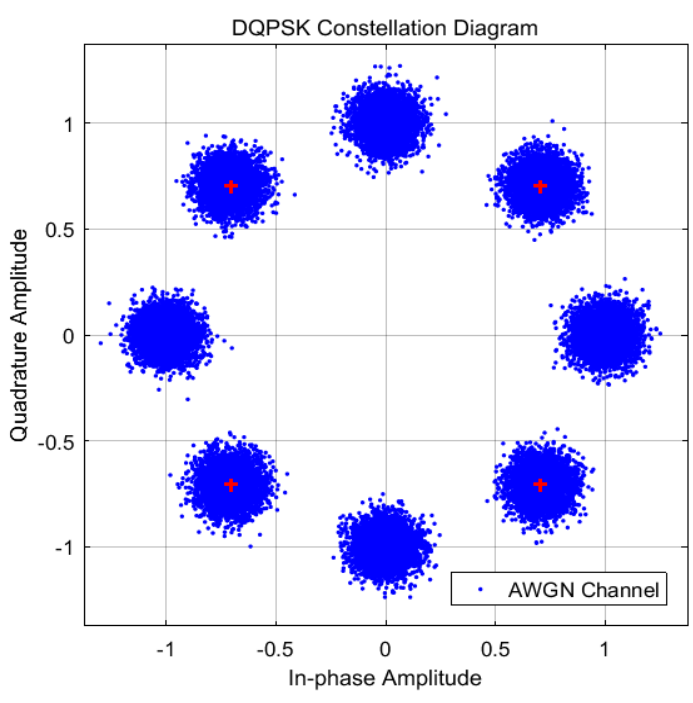

Figure 2. DQPSK Constellation Diagram

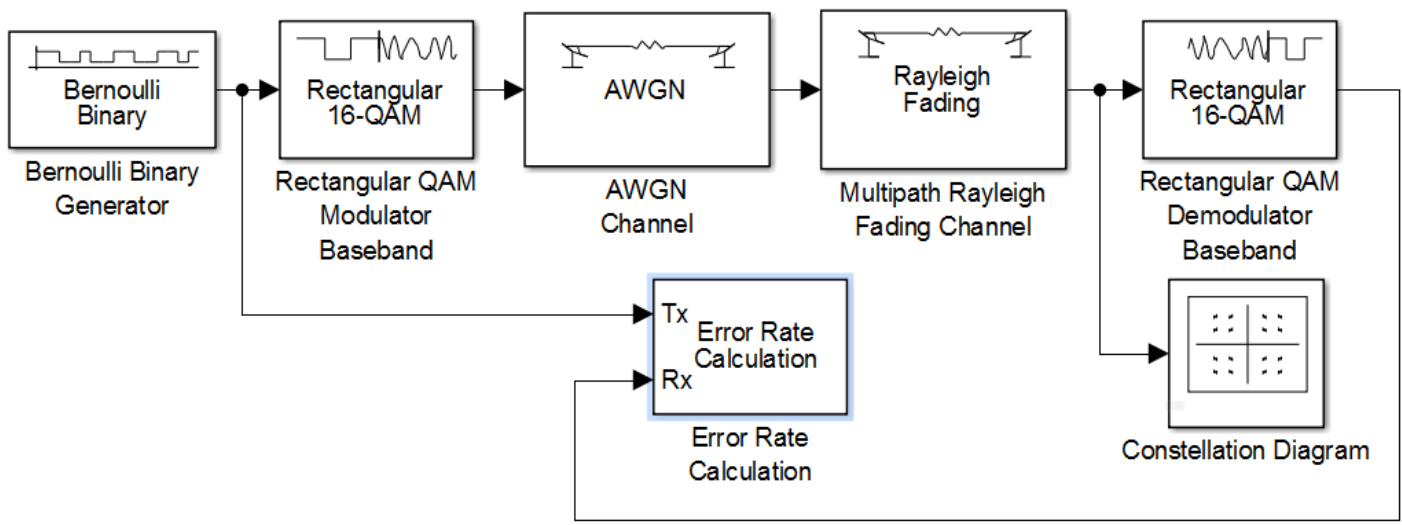

Figure 3 (a). 16-QAM signal model over Rayleigh fading \& AWGN channel 


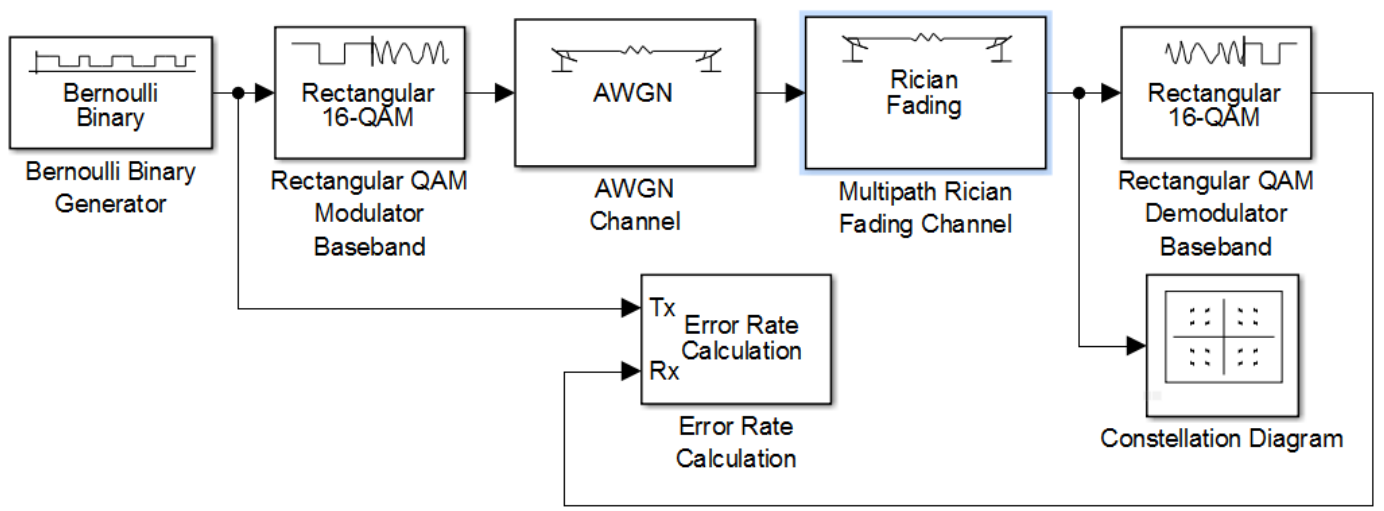

Figure. 3(b). 16-QAM signal model over Rician fading \&AWGN channel

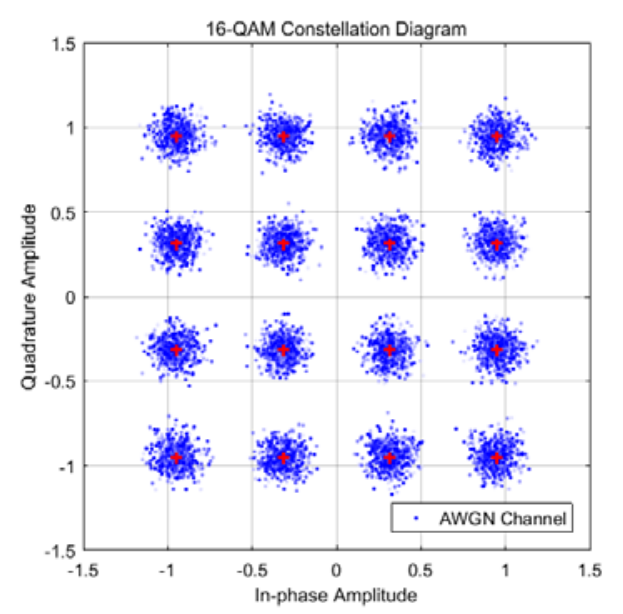

Figure 4. 16-QAM Constellation Diagram

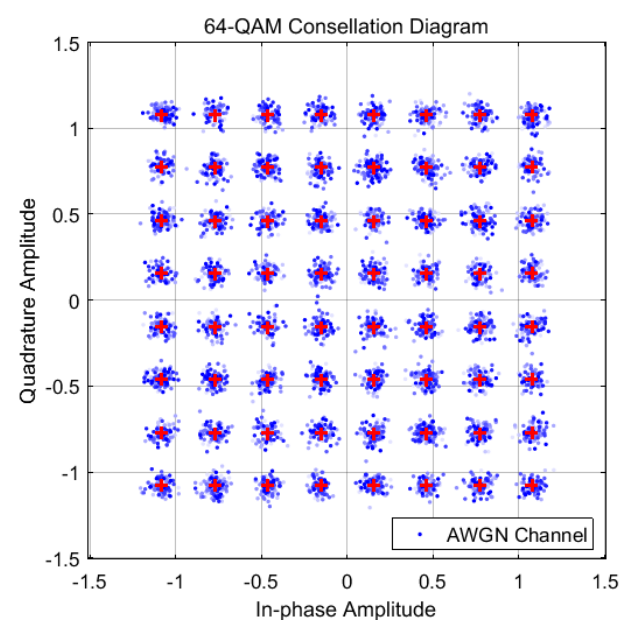

Figure 5. 64-QAM Constellation Diagram

Flowing, we have analyzed BER and SNR values of different modulation techniques in Rayleigh and Rician fading channels. Figure. 5 is the comparison of theoretical BER data of different modulation techniques over Rayleigh fading channel, and Figure. 7 is the comparison of theoretical BER data over different modulation techniques over Rician fading channel. From the simulation we can see that 64-QAM based system has the highest BER values over both Rayleigh and Rician fading channels. At the same time, QPSK based system has the lowest BER values over both Rayleigh and Rician fading channels. 


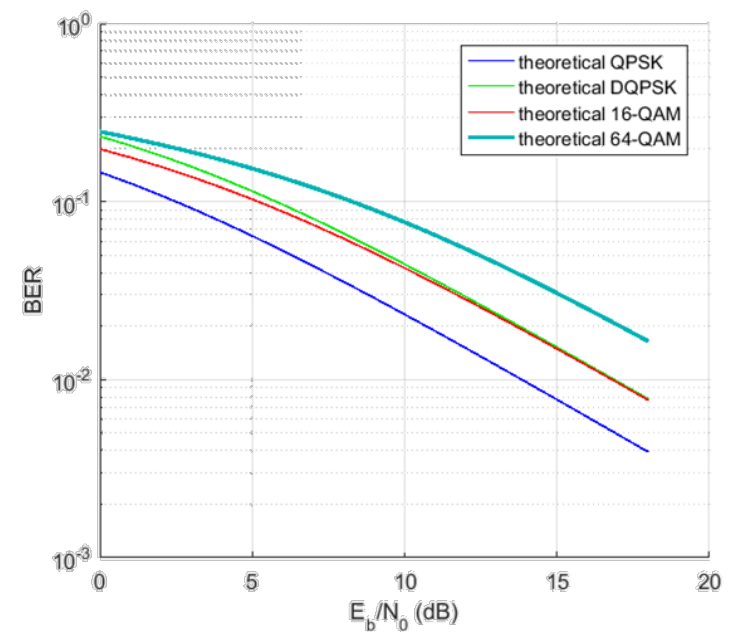

Figure 6. Theoretical BER over Rayleigh Channel

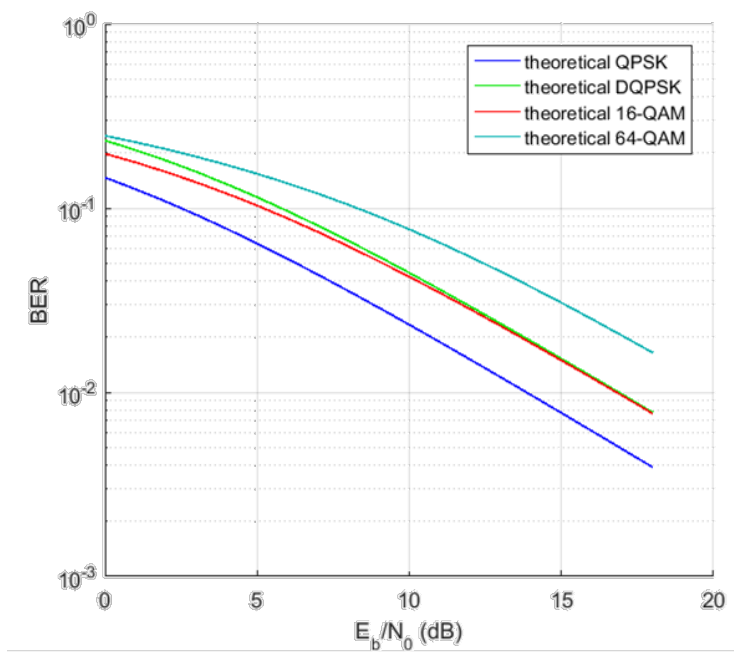

Figure.7 Theoretical BER over Rician Channel

In the Figure 8, we analyzed three modulation techniques symbol error rates (SNR) per bit. From the figure we can see that 64-QAM based system has the highest SNR value, and the QPSK has the lowest value.

At last, we use the BERTool in conjunction with Matlab Simulation functions to generate and analyze BER of Monte Carlo 16-QAM base system, and Figure 9 shows the result.

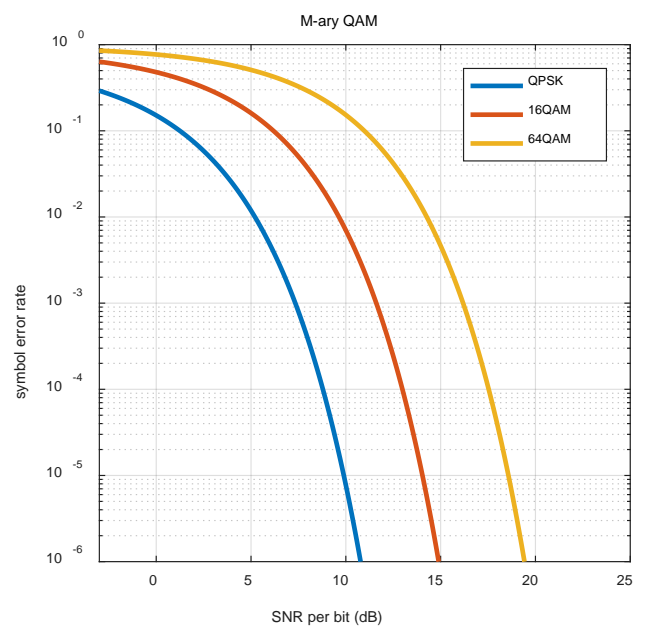

Figure 8. Bit Error Rate Analysis

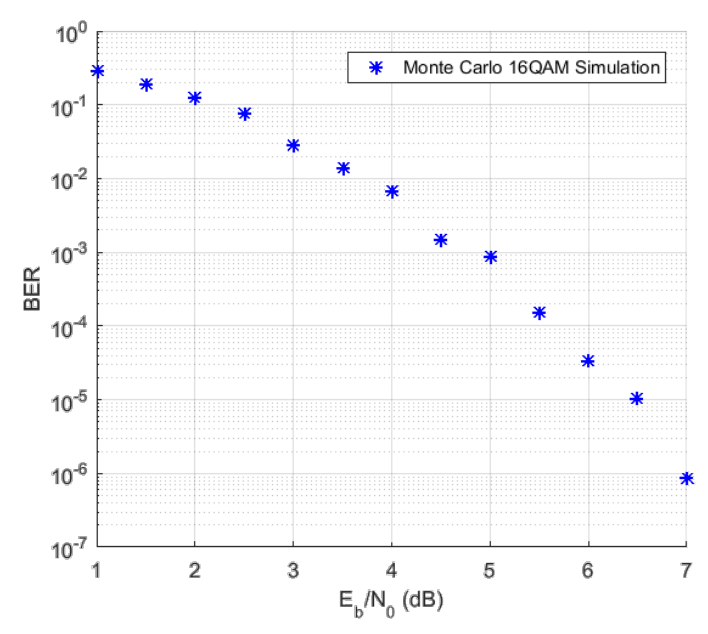

Figure 9. BER of Monte Carlo 16-QAM Simulation

\section{Conclusions}

In order to meet growing data demands on mobile networks from smart-phones and tablets, 4G communication systems has brought about development of high efficient and effective modulation techniques, such QPSK and QAM.

In this paper, we have discussed various digital modulation techniques such as QPSK, DQPSK, 16QAM and 64QAM, which have been used in the 4G communication systems. We did all the simulation to achieve a desired BER and SER over both AWGN channel and fading channels (Rayleigh and Rician Channels). And we also simulation studied constellations of different modulation themes. It can be concluded that 64-QAM can transmit more data but has highest BER and SER values, which means the higher order of QAM the less reliability, due to its high error rate. 


\section{References}

[1] Matthias Pätzold “Mobile Radio Channels”, Second Edition, 2011, A John Wiley \& Sons, Ltd., Publication.

[2] Ajay R. Mishra, Advanced Cellular NetworkPlanning and Optimisation, Wiley, 2007.

[3] Sayantan Acharya; Pulak Kabiraj; Debashis De, "Comparative analysis of different modulation techniques of LTE network", Proceedings of the 2015 Third International Conference on Computer, Communication, Control and Information Technology (C3IT)

[4] Jing Wang; Zhenhua Yu; "K. Delta-sigma modulation for digital mobile fronthaul enabling carrier aggregation of

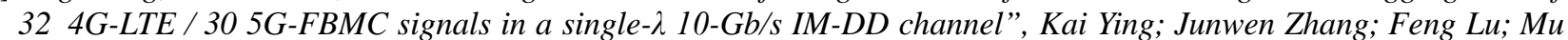
Xu; Gee-Kung Chang 2016 Optical Fiber Communications Conference and Exhibition (OFC)

[5] Charles U. Ndujiuba, Oluyinka Oni, Augustus E. Ibhaze. "Comparative Analysis of Digital Modulation Techniques in LTE 4G System”, Journal of Wireless Networking and Communication 2015, 5(2), P60-66.

[6] Swati Sharma, Harjit Singh "Comparison of Different Digital Modulation Techniques in LTE System using OFDM AWGN Channel: A Review”. International Journal of Computer Application, Vol 143, No.3 June 2016.

[7] Y. H. Yang et al., "Propagation Channel Modeling for Rayleigh and Rice Fading", Applied Mechanics and Materials, Vols. 687-691, pp. 3648-3651, 2014.

[8] S. O. Rice, "Statistical properties of a sine wave plus random noise,” Bell Syst. Tech. J., vol. 27, pp. 109-157, Jan. 1948.

[9] Papoulis, Athanasios; Pillai, S. (2001) Probability, Random Variables and Stochastic Processe.

[10] Nolan, Keith E., et al., "Modulation Scheme Classification for 4G Software Radio Wireless Networks," Proceedings of the International Conference on Signal Processing, Pattern Recognition and Applications (SPPRA, 2002), pp. 25-31.

[11] https://en.wikipedia.org/wiki.

[12] “Digital Baseband Modulation/Demodulation Techniques, Hands on Bit Error Calculations, Signal space analysis and receivers design” ENB7060 Telecommunications $B$. 